Cahiers $d u$ MONDE RUSSE

\section{Cahiers du monde russe}

Russie - Empire russe - Union soviétique et États indépendants

$62 / 4 \mid 2021$

Varia

\title{
Alexandre STROEV, dir., Les intellectuels russes à la conquête de l'opinion publique française. Une histoire alternative de la littérature russe en France de Cantemir à Gorki
}

\section{Catherine Depretto}

\section{OpenEdition}

\section{Journals}

Édition électronique

URL : https://journals.openedition.org/monderusse/12937

DOI : 10.4000/monderusse. 12937

ISSN : $1777-5388$

Éditeur

Éditions de l'EHESS

\section{Édition imprimée}

Date de publication : 1 décembre 2021

Pagination : 754-757

ISBN : 978-2-7132-2895-7

ISSN : $1252-6576$

Référence électronique

Catherine Depretto, «Alexandre STROEV, dir., Les intellectuels russes à la conquête de l'opinion publique française. Une histoire alternative de la littérature russe en France de Cantemir à Gorki », Cahiers du monde russe [En ligne], 62/4 | 2021, mis en ligne le 01 décembre 2021, consulté le 04 septembre 2022. URL: http://journals.openedition.org/monderusse/12937; DOI : https://doi.org/10.4000/monderusse. 12937

Ce document a été généré automatiquement le 4 septembre 2022.

Tous droits réservés 


\section{Alexandre STROEV, dir., Les intellectuels russes à la conquête de l'opinion publique française. Une histoire alternative de la littérature russe en France de Cantemir à Gorki}

\section{Catherine Depretto}

\section{RÉFÉRENCE}

Alexandre STROEV, dir., Les intellectuels russes à la conquête de l'opinion publique française. Une histoire alternative de la littérature russe en France de Cantemir à Gorki, Paris: Presses de la Sorbonne nouvelle, 2019, 368 p.

1 L'étude des relations culturelles entre la France et la Russie est un sujet de choix pour des raisons historiques bien connues qui tiennent, en particulier, à la place de la langue et de la littérature françaises dans l'essor intellectuel de la Russie au XVIII et au-delà. Cette thématique, déjà bien étudiée par la critique aussi bien en France qu'en Russie, s'est profondément renouvelée ces dernières décennies, grâce au développement généralisé des approches comparatistes, des problématiques de réception et de transferts culturels.

Professeur à l'université Sorbonne Nouvelle-Paris 3, spécialiste du XVIII ${ }^{\mathrm{e}}$ siècle de formation, Alexandre Stroev fait partie de ceux qui, depuis de nombreuses années, explorent de façon inventive ce vaste sujet. Un de ses premiers ouvrages était consacré aux Aventuriers des Lumières (1997) et plus récemment, dans La Russie et la France des Lumières (2017), paru sous sa direction, il n'hésite pas à faire revivre à côté des monarques et des philosophes, les figures de l'ombre que sont les espions. Dans Les intellectuels russes à la conquête de l'opinion française, la perspective est toujours celle des relations culturelles, des phénomènes de réception, mais avec une orientation de sociologie 
littéraire plus affirmée. Il est question, en effet, de mieux cerner les stratégies de conquête de l'opinion publique française de la part des auteurs russes et pas seulement de constater la présence de tel ou tel lien, d'où le sous-titre « Une histoire alternative de la littérature russe en France de Cantemir à Gorki ». L'ouvrage est issu d'un colloque qui s'est tenu à Paris en 2016. Il prenait la suite de deux manifestations scientifiques, ayant eu lieu respectivement à Tver' et à Tomsk et était le point d'aboutissement de projets de recherche franco-russes, soutenus par le CNRS et la Fondation russe de recherches fondamentales. Le livre s'ouvre sur un hommage à Alexander Yanushkevich (1944-2016), professeur à l'université de Tomsk, spécialiste de Žukovskij et associé à ce programme de recherches. Dans sa substantielle introduction, A. Stroev explicite la perspective adoptée et tout en présentant, comme il est coutume de le faire, les différentes contributions de l'ouvrage, élargit le sujet à d'autres exemples, concernant en priorité le xvIII siècle (Sumarokov, Fonvizin, Karamzin). ${ }^{1}$

3 Les intellectuels russes à la conquête de l'opinion française offre effectivement au lecteur un parcours atypique de l'histoire de la littérature russe des XVIII $^{\mathrm{e}}-\mathrm{XIX}^{\mathrm{e}}$ siècles principalement. Seuls deux articles (un sur Merežkovskij, l'autre sur Gor'kij) ont trait au $\mathrm{XX}^{\mathrm{e}}$ siècle. Si ce parcours est jalonné de figures célèbres, Kantemir, Krylov, Žukovskij, Puškin, Gogol', Turgenev, Dostoevskij, Tolstoj..., il égrène également des personnages moins connus, Sergej Ivanovič Turgenev (1790-1827), le benjamin des frères Turgenev, mort prématurément et mentionné ici pour son journal des années 1815-1816², Zinaida Volkonskaja, célèbre surtout pour ses salons ${ }^{3}$, le français Paul Lacroix, écrivant sous le pseudonyme de "Bibliophile Jacob» et auteur d'une biographie inachevée de Nicolas $\mathrm{I}^{\mathrm{er}} . .{ }^{4} \mathrm{Si}$ certains auteurs mobilisent énergie et relations pour essayer d'accéder à une certaine reconnaissance, d'autres, au contraire, ne semblent pas se soucier de bénéficier d'une quelconque notoriété. Parmi eux, on peut citer le cas en apparence paradoxal de Puškin, si pénétré de littérature française qu'il était surnommé "le Français » par ses condisciples du Lycée de Carskoe selo et qui pourtant n'aurait pas cherché à devenir célèbre en France. Parmi les hypothèses avancées : la difficulté que présente la traduction de ses vers, la piètre estime du poète pour la France de la Restauration et le fait qu'il lui était interdit de sortir de Russie (Olga Mouravieva, «Pourquoi Pouchkine n'a-t-il pas voulu conquérir le marché littéraire français? »). Un autre exemple bien connu est celui de Gogol' qui, lors de son séjour à Paris en 1836, n'a absolument pas cherché à fréquenter les milieux littéraires parisiens ou les cercles russes de la capitale. Ce refus de tout contact et a fortiori de toute quête de publicité contraste avec la stratégie déployée au même moment par Anatole Demidov, auteur d'un ouvrage écrit en français, Esquisse d'un voyage dans la Russie méridionale et la Crimée (1838), grâce auquel il espérait, en vain, acquérir la gloire (Claude de Grève, «Une programmation inexistante vs une stratégie 'insolente' : Nicolas Gogol et Anatoli Demidov à Paris dans les années 1830-1840»).

Dostoevskij fait aussi partie de ces auteurs qui ne cherchent pas à conquérir Paris : lors de son premier grand tour en Europe (1862), il se tient à l'écart, mais, à la différence de ses prédécesseurs, il rapporte de son voyage un témoignage peu flatteur sur l'Europe. Ses Notes d'hiver sur des impressions d'été comportent deux sujets particulièrement saisissants, la description du Londres industriel et de la misère ouvrière ( Baal») et surtout la dénonciation du Paris bourgeois (sur plusieurs chapitres). Karen Haddad $^{5}$ suggère de façon subtile que cette perception de la France, étouffée par l'esprit bourgeois, annonce en creux la réception future de son œuvre comme délivrant une "parole nouvelle». Cette présentation est, entre autres, celle de Melchior de Vogüé qui, dans Le Roman russe 
(1886), contre le naturalisme, «plaide en faveur d'un ressourcement de la littérature française auprès des forces vives de la Russie $»^{6}$.

5 Les exemples de Gogol' et de Dostoevskij nous font mesurer, par contraste, combien est différente l'attitude de Žukovskij lors de son séjour à Paris en 1827. Pensant sans doute à sa charge de précepteur de l'héritier au trône de Russie, il fait l'acquisition de nombreux ouvrages, déploie de grands efforts pour se pénétrer des courants d'idées de la capitale française, pour se familiariser avec son milieu intellectuel et politique, mais aussi scientifique, théâtral... ainsi qu'en témoignent les pages de son journal (Olga Lebedeva, Alexander Yanushkevich, "Comment le poète construit sa vie: le journal parisien de Vassili Joukovski (1827)»). Si Žukovskij se montre curieux de tout et très réceptif, tel n'est pas Nikolaj Greč dont les différents écrits, issus de ses voyages à l'étranger véhiculent une image stéréotypée et partiale de l'Europe et de la France, conforme à l'attente d'une certaine opinion publique russe (Alexander Sorotchan, «La diplomatie littéraire dans les Lettres de voyage de Nikolaï Gretch»). La collaboration entre la Revue encyclopédique et les Russes se révèle tout aussi problématique : ainsi après avoir sollicité le prince Vjazemskij, la rédaction de la Revue non satisfaite finit par refuser ses articles et se tourne, faute de mieux, vers Jakov Tolstoj, bientôt officiellement agent de la police secrète de Nicolas I ${ }^{\text {er }}$ (Ekaterina Larionova, «La Revue encyclopédique comme tribune littéraire et politique russe).

6 Finalement, malgré des liens nombreux entre les écrivains russes et la culture française, cette "histoire alternative" fait apparaître plus d'échecs que de réussites dans les tentatives de collaboration et de compréhension mutuelle. Cela s'applique au cas un peu à part du polonais Jan Potocki, célèbre aujourd'hui pour Le Manuscrit trouvé à Saragosse, mais qui, de son vivant, n'arrive pas à se faire reconnaitre aussi bien en France qu'en Russie $^{7}$. Il en va ainsi également de cette édition des fables de Krylov, associant texte en russe et traductions en français et en italien, qui n'a pas vraiment le résultat attendu (Andreï Dobrytsin, «Les premières traductions françaises des fables d'Ivan Krylov »). Quant à Turgenev, il doit se plier à de nombreuses exigences pour rendre conforme aux attentes du public français ses Eaux printanières ${ }^{8}$. Cet exemple met particulièrement bien en lumière l'imbrication des questions de réception et de traduction.

7 Si les pratiques du XIX en matière de traduction étaient loin des exigences actuelles, il est un auteur, Dostoevskij, dont l'œuvre, malgré tout l'intérêt qu'elle suscitait, a particulièrement souffert des libertés prises avec le texte d'origine ${ }^{9}$. Elena Galtsova analyse un exemple très intéressant, réunissant abusivement deux œuvres distinctes de Dostoevskij, Les Carnets du sous-sol et La Logeuse, sous un titre qui, paradoxalement, sonne juste, L'Esprit souterrain (1886). Elle étudie également les adaptations de ce texte, en particulier celle de Georges Bataille pour la radio en $1946^{10}$. Quant à l'œuvre de Lev Tolstoj, elle est également malmenée par les traducteurs français pour des raisons qui tiennent aux pratiques habituelles et à des raisons de censure, comme dans le cas, entre autres, de Résurrection, traduit par Théodore de Wyzewa (Alla Polosina, «Lev Tolstoï défiguré : censure et traductions ").

Pour la période de la fin du $\mathrm{xIX}^{\mathrm{e}}$ - début $\mathrm{du} \mathrm{xx}^{\mathrm{e}}$ siècle, on retrouve un même contraste entre un Merežkovskij qui déploie beaucoup d'efforts pour se faire connaître, en s'appuyant en particulier sur la médiation de Maurice Prozor ${ }^{11}$ et un Gor'kij, connu assez vite du public français, sans avoir spécialement contribué à cette popularité. Celle-ci tient à l'existence de traductions, mais est aussi favorisée par son image d'écrivain des va-nu-pieds et son aura d'opposant politique, persécuté par le régime tsariste ${ }^{12}$. 
9 Tout au long de ce parcours, on a vu l'importance de la composante politique pour éclairer les réactions de l'opinion publique française et pour comprendre le comportement des écrivains et intellectuels, russes ou français. Cette dimension apparait tout particulièrement dans l'exemple de Kantemir, qui ouvre le recueil : en acceptant de réfuter un pamphlet de Francesco Locatelli, il agit sans doute plus en polémiste qu'en écrivain (Francine-Dominique Liechtenham, "Le prince Cantemir et sa réfutation des Lettres moscovites de Francesco Locatelli »).

Comme souvent lorsque l'on a affaire à des articles issus d'un colloque, les contributions sont de nature différente et répondent chacune à leur manière à la thématique générale. Si certains sujets reprennent des épisodes bien connus, d'autres en revanche s'appuient sur des matériaux inédits et apportent des éclairages nouveaux. Dans certains cas, on a plus le sentiment de retrouver des études de réception qu'une analyse véritable des mécanismes de création d'une réputation dans une perspective sociologique. Sans doute était-il prématuré d'essayer de dresser une typologie visant à éclairer les comportements des différents acteurs de cette histoire alternative, en prenant en compte les trajectoires individuelles comme l'histoire des relations entre la France et la Russie (Alexandre Stroev fournit quelques pistes dans son introduction). S'il ne répond pas toujours parfaitement à l'ambition affichée, l'ouvrage n'en constitue pas moins un bel apport à l'étude des relations culturelles entre la France et la Russie.

\section{NOTES}

1. Alexandre Stroev, "'A nous deux, Paris' : pour une histoire alternative de la littérature russe en France », p. 11-50.

2. Nina Dmitrieva, «La mentalité russe et la civilisation française : les journaux parisiens de Sergueï Tourgueniev (1815-1816)».

3. Ekaterina Dmitrieva, «Entre Moscou, Paris et Rome: les salons de la princesse Zinaïda Volkonskaïa ".

4. Irina Staf, «L'Histoire de la vie et du règne de Nicolas $\mathrm{I}^{\text {er }}$ de Paul Lacroix (Bibliophile Jacob) : une œuvre de commande russe ».

5. Karen Haddad, «Impressions hivernales (et désobligeantes) d'un voyageur russe. Le 'A nous deux Paris' de Dostoïevski».

6. Pierre-Jean Dufief, «Le Roman russe de Vogüe et le dialogue des cultures », p. 281.

7. Dzianis Kandakou, « Jean Potocki à Paris : une célébrité manquée?»

8. Polina de Mauny, «La fortune française du roman d'Ivan Tourgueniev, Eaux printanières ».

9. Cf. "Dostoïevski», in Bernard Banoun, Yves Chevrel, Isabelle Poulin, dir., Histoire des traductions en langue française. Le XXe, Lagrasse : Verdier, 2019, p. 377-384.

10. Elena Galtsova, «D'un Esprit souterrain l'autre : de la première traduction des Carnets du soussol de Dostoïevski à la pièce de théâtre de Lenormand et la pièce radiophonique de Georges Bataille ".

11. Vadim Polonski, « Dmitri Merejkovski et le comte Maurice Prozor ».

12. Serge Rolet, « La découverte de Gorki en France au début des années 1900 ». 


\section{AUTEURS}

CATHERINE DEPRETTO

Paris Sorbonne 